T. Sekiguchi

Nagoya Math. J.

Vol. 82 (1981), 57-82

\title{
THE COINCIDENCE OF FIELDS OF MODULI FOR NON-HYPERELLIPTIC CURVES AND FOR THEIR JACOBIAN VARIETIES
}

\author{
TSUTOMU SEKIGUCHI
}

\section{Introduction}

The notion of fields of moduli introduced first by Matsusaka [8] has been developed by Shimura [12] exclusively in the area of polarized abelian varieties. Later Koizumi [7] gave an axiomatic treatment for the notion.

Geometrically, the notion of fields of moduli for a polarized abelian variety and for a curve may well be given by the residue field at the corresponding point on the moduli space, but the author thinks that there is no proof of it which is available for any characteristic case. Moreover, the expected fact seems to give a good approach to the investigation of the Torelli map from the moduli space of curves to that of principally polarized abelian varieties. In fact, in the present paper we will show the following fact:

The field of moduli for a non-hyperelliptic complete non-singular curve over a field of positive characteristic coincides with that of its canonically polarized jacobian variety.

The difficulty of this fact is on the inseparability, and so it is trivial for characteristic 0 .

In particular, when we restrict ourselves to the curves of genus $\leqq 3$, Oort-Ueno [11] showed that every principally polarized abelian variety of dimension $\leqq 3$ over an algebraically closed field is the polarized jacobian variety of a good curve. But, for curves of genus 3 , we can prove a stronger result:

For a principally polarized abelian variety $(X, \lambda)$ of dimension 3 over a field $k$, there exists a good curve over the same field $k$ whose polarized jacobian variety coincides with $(X, \lambda)$.

Received June 8, 1979.

Revised October 22, 1979. 
The main tool for proving these facts is the deformations of curves and of polarized abelian varieties.

In Section 1, we shall study the lifting problem about discrete valuation rings. The notion of fields of moduli which is used in the paper will be reviewed in Section 2 mainly following Koizumi [7]. Section 3 and 4 are devoted to investigating the compatibility of the specializations of a curve and of its jacobian variety, which will play an essential role in the proof of our main theorems. In the last two sections 5 and 6 , we shall give our main theorems.

The author would like to express his hearty thanks to Professor S. Koizumi for very useful conversations. In particular, the author was taught some problems about the fields of moduli for curves and for polarized abelian varieties.

\section{Notation}

Throughout the paper we mean by an abelian scheme a projective abelian scheme. For an abelian scheme $\mathfrak{X}$ over a noetherian scheme $S$ and for an invertible sheaf $\mathscr{L}$ on $\mathfrak{X}$, we denote by $\Lambda(\mathscr{L}): \mathfrak{X} \rightarrow \hat{\mathfrak{X}}$ the homomorphism from $\mathfrak{X}$ to the dual abelian scheme $\hat{X}$ of $\mathfrak{X}$ defined by the divisorial correspondence $\mu^{*} \mathscr{L} \otimes p_{1}^{*} \mathscr{L}^{-1} \otimes p_{2}^{*} \mathscr{L}^{-1}$ on $\mathfrak{X} \times_{s} \mathfrak{X}$, where $\mu: \mathfrak{X} \times_{s} \mathfrak{X}$ $\rightarrow \mathfrak{X}$ is the addition morphism and $p_{i}: \mathfrak{X} \times_{s} \mathfrak{X} \rightarrow \mathfrak{X}$ is the canonical projection for each $i=1,2$.

Let $X$ and $Y$ be preschemes over a prescheme $S$, and $f: X \rightarrow Y$ a morphism over $S$. Then for any point $s$ of $S$, we denote by $X_{s}$ (resp. $f_{s}$ ) the fibre prescheme $X \times{ }_{S} \operatorname{Spec}\left(k(s)\right.$ ) (resp. $f \times{ }_{S} \operatorname{Spec}(k(s))$ ) where $k(s)$ is the residue field at $s$ on $S$. In particular, for a pair $(\mathfrak{X}, \lambda)$ of an abelian scheme $\mathfrak{X}$ over $S$ and a homomorphism $\lambda: \mathfrak{X} \rightarrow \mathfrak{X}$, we put $(\mathfrak{X}, \lambda)_{s}=\left(\mathfrak{X}_{s}, \lambda_{s}\right)$ for a point $s$ of $S$.

Let $S$ be a noetherian scheme, and $\mathscr{C}$ a simple, proper $S$-scheme whose geometric fibres are irreducible curves. Then we denote by $(J(\mathscr{C}), \lambda(\mathscr{C}))$ the canonical polarized jacobian scheme over $S$ corresponding to $\mathscr{C}$ (cf. [9], Proposition 6.9). Moreover, for a trait $S$ and a good curve $\mathscr{C}$ over $S$, we use the same notation $(J(\mathscr{C}), \lambda(\mathscr{C}))$ as the canonical polarized jacobian scheme over $S$ corresponding to $\mathscr{C}$ (cf. [11]).

For an integral domain $R$, f.f. $(R)$ denotes the field of fractions of $R$. For a field $k, \bar{k}$ denotes an algebraic closure of $k$. 


\section{§1. Valuation ring with a given residue field}

First we shall start with some preliminaries from discrete valuation rings.

The following fact is well-known, so we state it here without any proof.

Theorem 1.1. Let $(A, \mathfrak{M})$ be a discrete valuation ring with residue field $k$. Let $k^{\prime}$ be any extension field of $k$. Then there exists a discrete valuation ring $A^{\prime}$ containing $A$, with maximal ideal $\mathfrak{M} A^{\prime}$ and with residue field $k^{\prime}$.

From this theorem, we can easily deduce

Corollary 1.2. Let $k$ be a field of characteristic $p>0$. Then there exists a discrete valuation ring $A$ of characteristic 0 with maximal ideal $p A$ and with residue field $k$.

The next proposition will be used as a technical tool in the proof of our main theorem.

Proposition 1.3. Let $K$ be a field and $L$ a finite algebraic extension of $K$. Let $\left(A, \mathfrak{M}_{A}\right)$ be a discrete valuation ring for $K$ and $\left(B, \mathfrak{M}_{B}\right)$ a discrete valuation ring for $L$ over $\left(A, \mathfrak{M}_{A}\right)$. Let $k^{\prime}$ and $\ell^{\prime}$ be finitely generated fields over the residue fields $k=A / \mathfrak{M}_{A}$ and $\ell=B / \mathfrak{M}_{B}$ such that $\ell^{\prime} \supset k^{\prime}$ and $\ell^{\prime}$ $=k^{\prime} \ell$. Then there exist finitely generated fields $K^{\prime}$ over $K$ and $L^{\prime}$ over $L$; and discrete valuation rings $\left(A^{\prime}, \mathfrak{M}_{A^{\prime}}\right)$ for $K^{\prime}$ and $\left(B^{\prime}, \mathfrak{M}_{B^{\prime}}\right)$ for $L^{\prime}$ with residue fields $k^{\prime}$ and $\ell^{\prime \prime}$, respectively, such that $B^{\prime}>A^{\prime}>A, B^{\prime}>B, \mathfrak{M}_{A^{\prime}}=$ $\mathfrak{M}_{A} A^{\prime}, L^{\prime}=K^{\prime} L$ and $\ell^{\prime \prime}$ is a finite algebraic extension of $\ell^{\prime}$. Moreover, if the algebraic closure of $k$ in $k^{\prime}$ is separable over $k$, we can choose $K^{\prime}$ so that the algebraic closure of $K$ in $K^{\prime}$ is separable over $K$.

Proof. First we assume that $k^{\prime}$ is purely transcendental over $k$, say $k^{\prime}=k\left(x_{1}, \cdots, x_{r}\right)$. Let $\pi_{A}: A\left[x_{1}, \cdots, x_{r}\right] \rightarrow k\left[x_{1}, \cdots, x_{r}\right]$ and $\pi_{B}: B\left[x_{1}, \cdots, x_{r}\right]$ $\rightarrow \ell\left[x_{1}, \cdots, x_{r}\right]$ be the canonical maps. We put $S=\pi_{A}^{-1}\left(k\left[x_{1}, \cdots, x_{r}\right]^{*}\right)$ and $S^{\prime}=\pi_{B}^{-1}\left(\ell\left[x_{1}, \cdots, x_{r}\right]^{*}\right)$, and we set

$$
A^{\prime}=S^{-1} A\left[x_{1}, \cdots, x_{r}\right] \text { and } B^{\prime}=S^{\prime-1} B\left[x_{1}, \cdots, x_{r}\right] .
$$

Then obviously these $A^{\prime}$ and $B^{\prime}$ satisfy our requiring properties.

Second, we assume that $k^{\prime}$ is algebraic over $k$; say $k^{\prime}=k\left(\alpha_{1}, \cdots, \alpha_{n}\right)$. Here we put $k_{i}=k\left(\alpha_{1}, \cdots, \alpha_{i}\right)$ and $\ell_{i}=\ell\left(\alpha_{1}, \cdots, \alpha_{i}\right)$ for $i=1,2, \cdots, n$. 
In particular, we put $k_{0}=k$ and $\ell_{0}=\ell$. Now we assume that we obtain discrete valuation rings $\left(A_{i}, \mathfrak{M}_{A_{i}}\right)$ and $\left(B_{i}, \mathfrak{M}_{B_{i}}\right)$ with $K_{i}=$ f.f. $\left(A_{i}\right), L_{i}=$ f.f. $\left(B_{i}\right), \quad k_{i}=A_{i} / \mathfrak{M}_{A_{i}}$ and $\ell_{i}^{\prime}=B_{i} / \mathfrak{M}_{B_{i}}$ such that $B_{i}>A_{i}>A, B_{i}>B$, $\mathfrak{M}_{A_{i}}=\mathfrak{M}_{A} A_{i}, L_{i}=L K_{i}$ and $\ell_{i}^{\prime}$ is a finite algebraic extension of $\ell_{i}$. Embedding $\ell^{\prime}$ and $\ell_{i}^{\prime}$ into the algebraic closure of $k$, we put $\ell_{i}^{\prime \prime}=\ell_{i}^{\prime} \ell^{\prime}$.

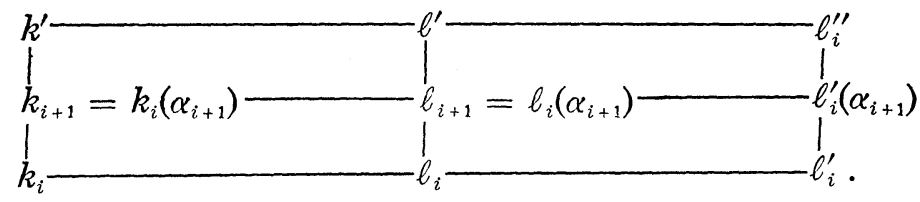

Let $F_{i}(X) \in k_{i}[X]$ (resp. $G_{i}(X) \in \ell_{i}[X]$ ) be the minimal polynomial of $\alpha_{i+1}$ with respect to $k_{i}$ (resp. $\left.\ell_{i}\right) ; F(X) \in A_{i}[X]$ (resp. $G(X) \in B_{i}[X]$ ) a monic polynomial such that $F \bmod \mathfrak{M}_{A_{i}}=F_{i}$ (resp. $\left.G \bmod \mathfrak{M}_{B_{i}}=G_{i}\right)$; and we put $A_{i+1}=A_{i}[X] /(F)$ and $K_{i+1}=$ f.f. $\left(A_{i+1}\right)$. Note that if $k_{i+1}$ is separable over $k_{i}$, so is $K_{i+1}$ over $K_{i}$. Let $F^{\prime}$ be an irreducible factor of $F$ in $B_{i}[X]$ such that $F^{\prime} \bmod \mathfrak{M}_{B_{i}}$ can be divided by $G_{i}$. Then we can easily see that $B_{i}[X] /\left(F^{\prime}\right)$ is a semilocal integral domain and $\left(\mathfrak{M}_{B_{i}}, G\right)$ is a maximal ideal of $B_{i}[X] /(F)$ over $\mathfrak{M}_{A_{i}}$ and $\mathfrak{M}_{B_{i}}$. Let $L_{i+1}=$ f.f. $\left(B_{i}[X] /\left(F^{\prime}\right)\right), \bar{B}_{i}=$ the derived normal ring of $B_{i}[X] /\left(F^{\prime}\right)$, and $\mathfrak{M}$ be a maximal ideal in $\bar{B}_{i}$ lying over the maximal ideal $\left(\mathfrak{M}_{B_{i}}, G\right)$ of $B_{i}[X] /(F)$. Then the localization at Obviously

$$
\begin{aligned}
& L_{i+1}=K_{i+1} L_{i}=K_{i+1} K_{i} L=K_{i+1} L, \\
& B_{i+1} / \mathfrak{M}_{B_{i+1}}=\bar{B}_{i} / \mathfrak{M} \supset\left(B_{i}[X] / F^{\prime}\right) /\left(\mathfrak{M}_{B_{i}}, G\right) \supset \ell_{i}^{\prime}, k_{i+1} .
\end{aligned}
$$

Thus we obtain inductively

$$
\begin{aligned}
& K_{0}=K \subset K_{1} \subset \cdots \subset K_{s} ; \quad L_{0}=L \subset L_{1} \subset \cdots \subset L_{s} ; \\
& A_{0}=A \subset A_{1} \subset \cdots \subset A_{s} \text { and } \quad B_{0}=B \subset B_{1} \subset \cdots \subset B_{s} .
\end{aligned}
$$

If we put $A^{\prime}=A_{s}, B^{\prime}=B_{s}, K^{\prime}=K_{s}$ and $L^{\prime}=L_{s}$, then obviously these satisfy our conditions. Moreover, by our constraction of them, if $k^{\prime}$ is saparably algebraic over $k$, so is $K^{\prime}$ over $K$.

Third, we suppose that $k^{\prime}$ is any finitely generated field over $k$. Let $k_{1}$ be the algebraic closure of $k$ in $k^{\prime}$; and we choose an intermediate 
field $k_{2}$ of $k^{\prime} / k_{1}$, so that $k^{\prime}$ is algebraic over $k_{2}$ and $k_{2}$ is purely transcendental over $k_{1}$. Then by the above considerations, we obtain fields $K_{1}$ over $K, K_{2}$ over $K_{1}, K^{\prime}$ over $K_{2}, L_{1}$ over $L, L_{2}$ over $L_{1}$ and $L^{\prime}$ over $L_{2}$; and discrete valuation rings $A^{\prime} \succ A_{2} \succ A_{1} \succ A$ for $K^{\prime} \supset K_{2} \supset K_{1} \supset K$ and $B^{\prime}>B_{2} \succ B_{1} \succ B$ for $L^{\prime} \supset L_{2} \supset L_{1} \supset L$ with residue fields $k^{\prime} \supset k_{2} \supset k_{1} \supset$ $k$ and $\ell^{\prime \prime}=B^{\prime} / \mathfrak{M}_{B^{\prime}} \supset \ell_{2}^{\prime}=B_{2} / \mathfrak{M}_{B_{2}} \supset \ell_{1}^{\prime}=B_{1} / \mathfrak{M}_{B_{1}} \supset \ell$, respectively, such that
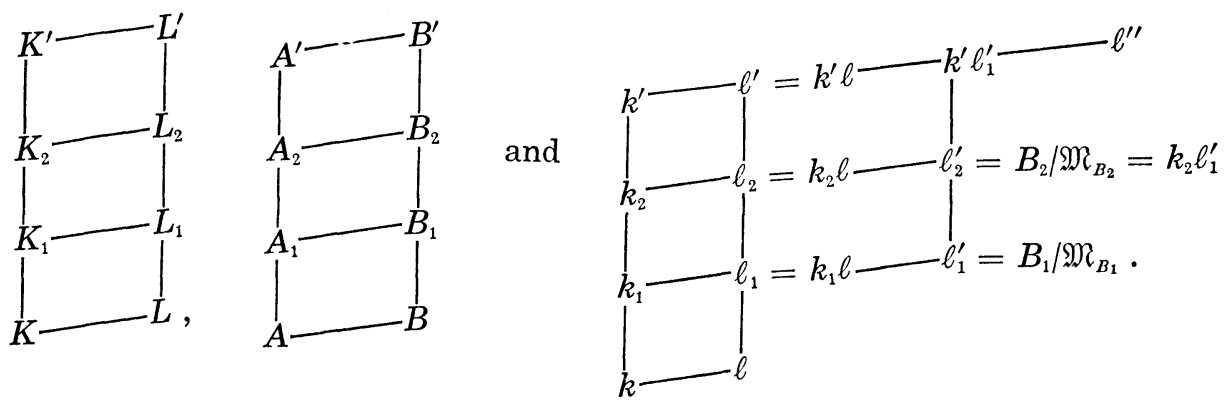

Moreover, by the above note, if $k_{1}$ is separable over $k$, so is $K_{1}$ over $K$. Last, we check that $K_{1}$ is algebraically closed in $K^{\prime}$. In fact, if $x \in K^{\prime}$ - $K_{1}$ is algebraic over $K_{1}, x \in K^{\prime}-K_{2}$. Hence $\left[K_{2}(x): K_{2}\right]>1$. But, by our construction of $K^{\prime}$, it has the unique extension $A^{\prime}$ of $A_{2}$, so $K_{2}(x)$ has also a unique extension $A_{2}^{\prime}$ of $A_{2}$, which is given by the integral closure of $A_{2}$ in $K_{2}(x)$. Therefore,

$$
\left[K_{2}(x): K_{2}\right]=\mathrm{e}\left(A_{2}^{\prime} / A_{2}\right) \mathrm{f}\left(A_{2}^{\prime} / A_{2}\right)=\mathrm{f}\left(A_{2}^{\prime} / A_{2}\right)>1,
$$

where e $\left(A_{2}^{\prime} / A_{2}\right)=$ the ramification index of $A_{2}^{\prime} / A_{2}$ and $\mathrm{f}\left(A_{2}^{\prime} / A_{2}\right)=$ the residual degree of $A_{2}^{\prime}$ over $A_{2}$. Hence there exists an element $z$ in $A_{2}^{\prime} \cap K_{1}(x)$ such that $z \bmod \mathfrak{M}_{A^{\prime}} \notin k_{1}$. This implies that $z$ is not algebraic over $K_{1}$, which is absurd.

Q.E.D.

Lemma 1.4. Let $A$ be a discrete valuation ring for a field $K$, and $\hat{K}$ the completion of $K$ with respect to the valuation. Assume $A$ is a Nagata ring; that is, for any finite algebraic extension $M$ of $K$, the integral closure of $A$ in $M$ is a finite A-module. Then the algebraic closure $\bar{K}$ of $K$ in $\hat{K}$ is separable over $K$.

Proof. Let $L$ be any finite extension over $K$; $\left\{v_{i}\right\}_{1 \leqq i \leqq s}$ a complete system of extensions over $L$ of the valuation given by $A$; and $\hat{L}_{i}$ the 
completion of $L$ for $v_{i}$. Then, since $A$ is a Nagata ring, we obtain the canonical ring-isomorphism

$$
\hat{K} \otimes_{K} L \simeq \prod_{i=1}^{s} L_{i}
$$

(cf. [2], Chap. 6, $\S 8, n^{\circ} 5$, Corollaire 3). Therefore $\bar{K} \otimes_{K} L$ has no nontrivial nilpotents, and we see the separability of $\bar{K} / K$ (cf. [1], Chap. 8, $\S 7, n^{\circ} 3$, Théorème 1 ).

Q.E.D.

\section{§2. Fields of moduli}

We shall quote here the notion of fields of moduli from Koizumi [7].

Definition 2.1 ([7], Definition 1.0.). Let $K$ be a field; $\Omega$ a universal domain containing $K$; and $\subseteq$ a collection of geometric objects $S, S^{\prime}, \cdots$, together with the following three laws:

i) Given an object $S$ and a subfield $L$ of $\Omega$ containing $K$, it is defined whether or not $L$ is a field of rationality for $S$. (When $L$ is a field of rationality for $S$, we say that $S$ is rational over $L$.)

ii) Given two objects $S$ and $S^{\prime}$, it is defined whether or not $S$ is isomorphic to $S^{\prime}$ (noted by $S \sim S^{\prime}$ ). We call this isomorphism the geometric isomorphism in $\subseteq$.

iii) Given an object $S$ rational over $L$ and a field $L^{\prime}$ with an injection $L \smile L^{\prime}$, the tensor product $S \otimes_{L} L^{\prime}$ is defined in $\varsigma$.

Such $\subseteq$ is called an $F M$-system over $K$ and an object $S$ in $\varsigma$ is called an FM-structure, when the pair $(\Im, K)$ satisfies the following conditions:

$\mathrm{fm}$ i) Under the notation in iii), $S \otimes_{L} L^{\prime}$ is rational over $L^{\prime}$. Moreover, if the injection $L \smile L^{\prime}$ is nothing but the identity, $S \otimes_{L} L^{\prime} \sim S$.

$\mathrm{fm}$ ii) The geometric isomorphism $\sim$ is an equivalence relation.

fm iii) Given $S$ and $S^{\prime}$ in $\subseteq$ rational over $L$ and $M$, respectively, we have

1) $S \otimes_{L} L=S$.

2) $S \sim S^{\prime}$ implies $S \otimes_{L} L^{\prime} \sim S^{\prime} \otimes_{M} L^{\prime}$ for a field $L^{\prime}$ with an injection $L M \subset L^{\prime}$.

3) $\left(S \otimes_{L} L^{\prime}\right) \otimes_{L^{\prime}} L^{\prime \prime}=S \otimes_{L} L^{\prime \prime}$ for fields $L^{\prime}$ and $L^{\prime \prime}$ with injections $L$ $\hookrightarrow L^{\prime} \lessdot L^{\prime \prime}$.

When $K$ is a prime field, we call $\subseteq$ simply an FM-system.

Now, let $\subseteq$ be an $F M$-system over a field $K$ and $S$ be an $F M$-structure in $\subseteq$ rational over $L$. For an isomorphism $\sigma$ over $K$ from $L$ to 
another field $L^{\prime}$ over $K$, we denote $S^{\sigma}=S \otimes_{L} L^{\prime}$.

Moreover we denote by FR $(S)$ the set consisting of all fields of rationality for all $S^{\prime}$ geometrically isomorphic to $S$.

Definition 2.2. A field $k_{S}$ containing $K$ is called a field of moduli for $S$ over $K$ (or simply a field of moduli for $S$ ), if it satisfies the following two conditions:

FM 1) $k_{S}=\cap L^{\prime}$ where $L^{\prime}$ runs over FR $(S)$.

FM 2) For any automorphism $\sigma \in \operatorname{Aut}(\Omega / K), S \sim S^{\sigma}$ if and only if the restriction of $\sigma$ on $k_{S}$ is the identity.

The existence of fields of moduli was showed for some important cases by Matsusaka, Shimura and Koizumi. Here we shall refer to two such cases, i.e., to curves and to polarized abelian varieties for latter use.

Let $\mho_{g}$ be the family of complete non-singular curves of genus $g$. For a curve $C$ in $\widetilde{C}_{g}$, as is known, we define the field of rationality for $C$ as the subfield of the rational function field $K(C)$, over which $C$ is defined and $K(C)$ is a regular extension. Obviously, it is given by the global sections $\Gamma\left(C, \mathcal{O}_{C}\right)$ (cf. EGA II, Corollaire (7.3.11)). Let $C$ and $C^{\prime}$ be two curves in $\widetilde{夭}_{g}$ rational over $k$ and $k^{\prime}$, respectively. Then we define $C \otimes_{k} l$ $=C \times_{\text {spec }(k)} \operatorname{Spec}(\ell)$ for any extension field $\ell$ over $k$. Moreover we define the geometric isomorphisms by

$C \sim C^{\prime}$ if and only if there exist a field $K$ containing both

$k$ and $k^{\prime}$ and a $K$-isomorphism $C \otimes_{k} K \simeq C^{\prime} \otimes_{k^{\prime}} K$.

Under these notations, we obtain

Theorem 2.3. $\mathfrak{夭}_{g}$ is an FM-system and there exists a field of moduli $k_{C}$ for any $C$ in $\mathfrak{F}_{g}$.

(cf. e.g., [7], Corollary 3.2.2.)

Next, let $X$ be an abelian scheme over a scheme $S$. Then we call a homomorphism $\lambda: X \rightarrow \hat{X}$ a polarization of $X$ if, for any geometric point $s \in S$, there exists an ample invertible sheaf $L$ on $X_{s}$ such that $\lambda_{s}=\Lambda(L)$.

Let $\mathfrak{B O}_{g, d}$ be the family of abelian varieties of dimension $g$ with a polarization of degree $d$. For an polarized abelian variety $(X, \lambda)$ in $\mathfrak{P R}_{g, d}$ over a field $k$, the field of rationality is given by $k$. Let $(X, \lambda)$ and $\left(X^{\prime}, \lambda^{\prime}\right)$ be two polarized abelian varieties in $\mathfrak{B A}_{g, d}$ over $k$ and $k^{\prime}$, respectively. Then the geometrical isomorphy of them is defined in the same way as in the case of curves. That is, 
$(X, \lambda) \sim\left(X^{\prime}, \lambda^{\prime}\right)$ if and only if there exists a field $K$ containing both $k$ and $k^{\prime}$ and an isomorphism $(X, \lambda) \otimes_{k} K \simeq\left(X, \lambda^{\prime}\right) \otimes_{k^{\prime}} K$.

Similarly we obtain

TheOREM 2.4. $\mathfrak{P O}_{g, d}$ is an FM-system and there exists a field of moduli for any object of $\mathfrak{B A}_{g, d}$.

(cf. [7], Theorem 2.2, Theorem 3.2 and Appendix to this paper, Proposition A3.)

\section{§3. Infinitesimal Torelli theorem}

Let $X$ be a complete non-singular curve rational over a field $k$, not necessarily having $k$-rational point, or a projective non-singular variety over $k$ having a $k$-rational point. Then there exists the Picard variety $J=\mathrm{Pic}_{X / k}^{\tau}$ of $X$ for both the cases. In these cases, we have a canonical isomorphism

$$
H^{1}\left(X, \mathcal{O}_{X}\right) \stackrel{\sim}{\longrightarrow} H^{0}\left(J, \check{\Omega}_{J}\right),
$$

where $\Omega_{J}$ is the sheaf of Kähler differential forms on $J$ and $\check{\Omega}_{J}$ is the dual sheaf of $\Omega_{\mathrm{J}}$.

If $C$ is a complete non-singular curve rational over a field $k$, and if we put $(J, \lambda)=(J(C), \lambda(C))$, then we can define the canonical isomorphism

$$
H^{1}\left(J, \mathcal{O}_{J}\right) \stackrel{\sim}{\longrightarrow} H^{1}\left(C, \mathcal{O}_{C}\right)
$$

by the commutative diagram

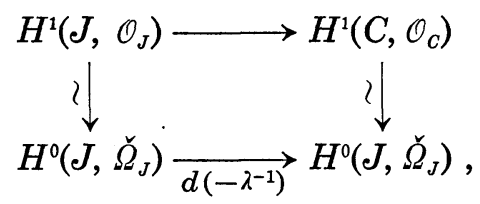

where the vertical arrows are given by (3.1). In particular, if $C$ has a $k$-rational point, we can embed $C$ into $J$, which we denote by $\iota: C \smile J$. Then the above isomorphism is equal to

$$
\iota^{*}: H^{1}\left(J, \mathcal{O}_{J}\right) \stackrel{\sim}{\longrightarrow} H^{1}\left(C, \mathcal{O}_{C}\right) .
$$

Therefore, since $\check{\Omega}_{J} \simeq \mathcal{O}_{J} \otimes_{k} H^{0}\left(J, \check{Q}_{J}\right)$, we have the canonical isomorphism

$$
H^{1}\left(J, \check{\Omega}_{J}\right) \simeq H^{1}\left(J, \mathcal{O}_{J}\right) \otimes H^{0}\left(J, \check{\Omega}_{J}\right) \simeq H^{1}\left(C, \mathcal{O}_{C}\right) \otimes H^{1}\left(C, \mathcal{O}_{c}\right)
$$


according to (3.1) and (3.2). Hence, passing through Serre duality, the cup product

$$
H^{0}\left(C, \Omega_{C}\right) \otimes H^{0}\left(C, \Omega_{C}\right) \longrightarrow H^{\circ}\left(C, \Omega_{C}^{\otimes 2}\right)
$$

defines the canonical map

$$
j: H^{1}\left(C, \check{\Omega}_{C}\right) \longrightarrow H^{1}\left(J, \check{\Omega}_{J}\right) .
$$

In particular, if there exists a natural embedding $\iota: C \subset J$, then we have the exact sequence

$$
0 \longrightarrow \mathscr{N}_{C / J} \longrightarrow \iota^{*} \Omega_{J} \stackrel{\iota^{*}}{\longrightarrow} \Omega_{C} \longrightarrow 0
$$

where $\mathscr{N}_{C / J}$ is the conormal sheaf, and we can easily see that the map (3.4) is nothing but the homomorphism

$$
H^{1}\left(\left(\iota^{*}\right)^{\vee}\right): H^{1}\left(C, \check{\Omega}_{C}\right) \longrightarrow H^{1}\left(C, \iota^{*} \check{\Omega}_{J}\right) \stackrel{\sim}{\longrightarrow} H^{1}\left(J, \check{\Omega}_{J}\right)
$$

Under these notations, we can rewrite Noether's theorem:

Proposition 3.1. Let $C$ be a complete non-singular curve of genus $g \geqq 2$, and $J$ the jacobian variety of $C$. Then the following are equivalent:

(i) $C$ is non-hyperelliptic.

(ii) The cup product

$$
H^{0}\left(C, \Omega_{C}\right) \otimes H^{0}\left(C, \Omega_{C}\right) \longrightarrow H^{0}\left(C, \Omega_{C}^{\otimes 2}\right)
$$

is surjective.

(iii) The map $j: H^{1}\left(C, \check{\Omega}_{C}\right) \rightarrow H^{1}\left(J, \check{\Omega}_{J}\right)$ is injective.

(iv) $\operatorname{dim} H^{0}\left(C, \mathscr{N}_{C / J}\right)=g$.

(v) $\operatorname{dim} H^{1}\left(C, \mathscr{N}_{C / J}\right)=g^{2}-3 g+3$.

Let $\left(A, \mathfrak{M}_{A}\right)$ and $\left(B, \mathfrak{M}_{B}\right)$ be artinian local rings, $f: B \rightarrow A$ a small surjection with $\operatorname{Ker} f=I$. Put $k=A / \mathfrak{M}_{A}, S=\operatorname{Spec}(A), S^{\prime}=\operatorname{Spec}(B)$ and $S_{0}=\operatorname{Spec}(k)$. Then we obtain the canonical closed immersions $S_{0} \longrightarrow S$ $\longrightarrow S^{\prime}$. Let $C \rightarrow S$ be a given smooth projective curve over $S, C^{\prime} \rightarrow S^{\prime}$ a given lifting of $C$ over $S^{\prime}$, and put $C_{0}=C \times{ }_{s_{0}} S$. Then obviously $\left(J^{\prime}, \lambda^{\prime}\right)=\left(J\left(C^{\prime}\right), \lambda\left(C^{\prime}\right)\right)$ is a lifting over $S^{\prime}$ of $(J, \lambda)=(J(C), \lambda(C))$ with $\left(J_{0}, \lambda_{0}\right)=\left(J\left(C_{0}\right), \lambda\left(C_{0}\right)\right)$ as the special fibre.

Now we put 


$$
\begin{array}{r}
\mathscr{L}\left(C ; S \longrightarrow S^{\prime}\right)=\left\{\left(C^{\prime \prime}, \phi\right) \mid \begin{array}{l}
C^{\prime \prime}: \text { projective simple curve over } S^{\prime} \\
\text { and an isomorphism } \phi: C^{\prime \prime} \times{ }_{S^{\prime}} S \widetilde{\rightarrow}
\end{array}\right\} \\
/\left\{\begin{array}{l}
\text { isomorphisms which induces } \\
\text { the identity on } C
\end{array}\right\}
\end{array}
$$

and

$$
\begin{aligned}
\mathscr{L}\left(J ; S \longrightarrow S^{\prime}\right)=\left\{\left(J^{\prime \prime}, \psi\right) \mid \begin{array}{l}
J^{\prime \prime}: \text { abelian scheme over } S^{\prime} \text { and an } \\
\text { isomorphism } \psi: J^{\prime \prime} \times{ }_{S^{\prime}} S \Im J
\end{array}\right\} \\
/\left\{\begin{array}{l}
\text { isomorphisms which induces } \\
\text { the identity on } J
\end{array}\right\} .
\end{aligned}
$$

Then Grothendieck's argument (cf. SGA 1, Exposé III) implies that these are principal homogeneous spaces under the additive groups

$$
H^{1}\left(C_{0}, \check{\Omega}_{C_{0} / S_{0}}\right) \otimes_{k} I \text { and } H^{1}\left(J_{0}, \check{\Omega}_{J_{0} / S_{0}}\right) \otimes_{k} I,
$$

respectively. Therefore, if we fix base points of these spaces, say $C^{\prime}$ and $J^{\prime}$, then we obtain isomorphisms

$$
\begin{aligned}
& H^{1}\left(C_{0}, \check{\Omega}_{C_{0} / S_{0}}\right) \otimes_{k} I \stackrel{\sim}{\longrightarrow}\left(C ; S \longrightarrow S^{\prime}\right), \\
& H^{1}\left(J_{0}, \check{\Omega}_{J_{0} / S_{0}}\right) \otimes_{k} I \longrightarrow \mathscr{L}\left(J ; S \longrightarrow S^{\prime}\right)
\end{aligned}
$$

such that $0 \mapsto C^{\prime}$ and $0 \mapsto J^{\prime}$. Moreover, let

$$
\mathscr{J}: \mathscr{L}\left(C ; S \smile S^{\prime}\right) \longrightarrow \mathscr{L}\left(J ; S \smile S^{\prime}\right)
$$

be the map which assigns to a curve $C^{\prime \prime}$ in $\mathscr{L}\left(C ; S \hookrightarrow S^{\prime}\right)$ its Picard scheme.

Under these notations, an easy calculation leads us to

Proposition 3.2. The following diagram is commutative:

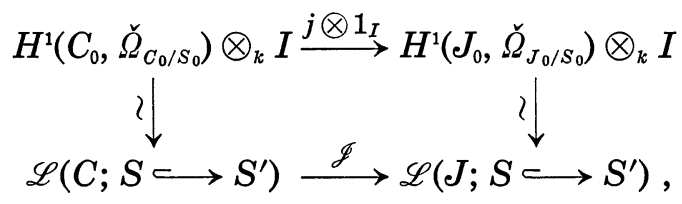

where the vertical arrows are those given by (3.6) and $j$ is the map (3.4).

In the rest of this section, we shall discuss the base change map.

Let $A, A^{\prime}$ and $B^{\prime}$ be artinian local rings; $f: A^{\prime} \rightarrow A$ a small surjection with $\operatorname{Ker} f=I$; and $g: A^{\prime} \rightarrow B^{\prime}$ a local homomorphism. We put $B=B^{\prime}$ 
$\otimes_{A^{\prime}} A, I^{\prime}=\operatorname{Ker}\left(f^{\prime}=f \otimes B^{\prime}: B^{\prime} \rightarrow B\right)$ and $k=A / \mathfrak{M}_{A}$. Then we obtain the following commutative diagram with exact horizontal lines.

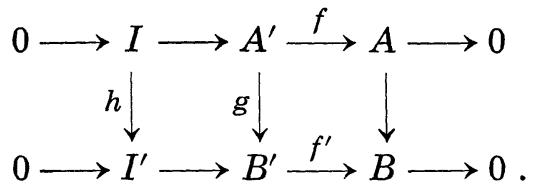

Obviously $I^{\prime 2}=(0)$. Now let $X$ be a smooth scheme over $\operatorname{Spec}(A)$ with special fibre $X_{0}=X \otimes_{A} k$, and $X^{\prime}$ a lifting of $X$ over $\operatorname{Spec}\left(A^{\prime}\right)$. Then $X_{B^{\prime}}^{\prime}=X^{\prime} \otimes_{A^{\prime}} B^{\prime}$ is also a lifting of $X_{B}=X \otimes_{A} B$ over $\operatorname{Spec}\left(B^{\prime}\right)$, and we get the base change map

$$
\otimes_{A^{\prime}} B^{\prime}: \mathscr{L}\left(X ; \operatorname{Spec}(A) \longrightarrow \operatorname{Spec}\left(A^{\prime}\right)\right) \longrightarrow \mathscr{L}\left(X_{B} ; \operatorname{Spec}(B) \longrightarrow \operatorname{Spec}\left(B^{\prime}\right)\right)
$$

assigning $X_{B^{\prime}}^{\prime}$ to $X^{\prime}$.

On the other hand, since $\check{\Omega}_{X / A}$ is a locally free $\mathcal{O}_{X}$-module of finite rank, $\check{\Omega}_{X_{B} / B}$ is canonically isomorphic to $\check{\Omega}_{X / A} \otimes_{A} B$. Hence we obtain the canonical isomorphism

$$
H^{1}\left(X_{0}, \check{\Omega}_{X_{B} / B} \otimes_{B} I^{\prime}\right) \simeq H^{1}\left(X_{0}, \check{\Omega}_{X_{0} / k}\right) \otimes_{k} I^{\prime} .
$$

So we get easily

Lemma 3.3. We fix a base element $X^{\prime}$ in $\mathscr{L}\left(X ; \operatorname{Spec}(A) \longrightarrow \operatorname{Spec}\left(A^{\prime}\right)\right)$ and let

$$
H^{1}\left(X_{0}, \check{\Omega}_{X_{0} / k}\right) \otimes_{k} I \stackrel{\sim}{\longrightarrow}\left(X ; \operatorname{Spec}(A) \smile \operatorname{Spec}\left(A^{\prime}\right)\right)
$$

and

$$
H^{1}\left(X_{0}, \check{\Omega}_{X_{0} / k}\right) \otimes_{k} I \stackrel{\sim}{\longrightarrow} \mathscr{L}\left(X_{B} ; \operatorname{Spec}(B) \smile \operatorname{Spec}\left(B^{\prime}\right)\right)
$$

be the canonical isomorphisms such that $0 \mapsto X^{\prime}$ and $0 \rightarrow X_{B^{\prime}}^{\prime}$, respectively. Then the following diagram commutes:

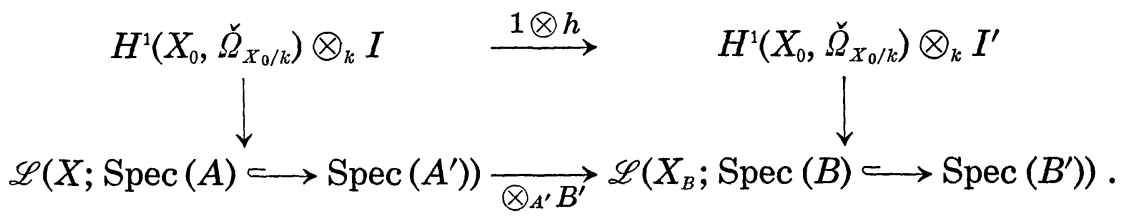




\section{§4. Specializations of a curve and of its jacobian variety}

The compatibility of stable reductions of a curve and of its jacobian variety was showed by Deligne-Mumford [3]. For good reductions, the same result is obtained over any discrete valuation rings. This result was given by Oort-Ueno [11, Lemma 6] for good curves.

Here we start this section with Oort-Ueno's result adding fields of rationality.

Proposition 4.1 (Oort-Ueno). Let $A$ be a discrete valuation ring with residue field $k=A / \mathfrak{M}_{A}$ and $K=$ f.f. $(A)$. Let $\mathfrak{X}$ be an abelian scheme over $S=\operatorname{Spec}(A)$ with a principal polarization $\lambda$, and $C$ a good curve (in the sense of [11]) over $K$ of genus $g \geqq 2$ having an isomorphism

$$
\phi_{0}:(J(C), \lambda(C)) \stackrel{\sim}{\longrightarrow}(\mathfrak{X}, \lambda) \otimes_{A} K .
$$

Then, there exist a good curve $\mathscr{C}$ over $S$ with $C$ as the generic fibre and an isomorphism

$$
\phi:(J(\mathscr{C}), \lambda(\mathscr{C})) \stackrel{\sim}{\longrightarrow}(\mathfrak{X}, \lambda)
$$

such that $\phi \otimes_{A} K=\phi_{0}$.

Proof. If $K$ is sufficiently large, $C$ can be embedded into $J(C)$ in a natural way and $\lambda=\Lambda(L)$ for some invertible sheaf $\mathscr{L}$ on $\mathfrak{X}$. Therefore, our assertion in this case is included in [11, Lemma 6].

The general case can be reduced to the above case quite in the same way as the proof of Theorem 2.5 in [3]. In fact, $C \otimes_{K} K^{\prime}$ and $(\mathfrak{X}, \lambda) \otimes_{K} K^{\prime}$ satisfy the above conditions for a suitable finite extension $K^{\prime}$ of $K$. Let $A^{\prime}$ be the localization at some maximal ideal of the integral closure of $A$ in $K^{\prime}$, and put $S^{\prime}=\operatorname{Spec}\left(A^{\prime}\right)$. Then $A^{\prime}$ is also a discrete valuation ring and $S^{\prime}$ is faithfully flat over $S$. We put $S^{\prime \prime}=S^{\prime} \times{ }_{S} S^{\prime}$. Moreover we denote by $\eta$ the generic point of $S$ and by $C^{\prime}$ and $C^{\prime \prime}$ the inverse images of $C$ on $S_{\eta}^{\prime}$ and on $S_{\eta}^{\prime \prime}$, respectively.

Then, by the above Oort-Ueno's result, there exist a curve $\mathscr{C}^{\prime}$ over $S^{\prime}$ having $C^{\prime}$ as generic fibre and an isomorphism $\phi^{\prime}:\left(J\left(\mathscr{C}^{\prime}\right), \lambda\left(\mathscr{C}^{\prime}\right)\right) \widetilde{\rightarrow}\left(\mathfrak{X}^{\prime}, \lambda^{\prime}\right)$ such that $\phi_{K^{\prime}}^{\prime}=\phi_{0} \otimes_{K} K^{\prime}$, where $\left(\mathfrak{X}^{\prime}, \lambda^{\prime}\right)=(\mathfrak{X}, \lambda) \otimes_{A} A^{\prime}$.

Now let

$$
S^{\prime \prime} \underset{p_{2}}{\stackrel{p_{1}}{\longrightarrow}} S^{\prime} \stackrel{p}{\longrightarrow} S
$$


be the canonical projections. Since $\mathscr{C}_{\eta}^{\prime}=C^{\prime}=C \otimes_{K} K^{\prime}$, it carries a descent datum with respect to $p$, i.e., an isomorphism $f_{\eta}: p_{1}^{*}\left(\mathscr{C}^{\prime}\right)_{\eta} \widetilde{\rightarrow} p_{2}^{*}\left(\mathscr{C}^{\prime}\right)_{\eta}$. So, by the same argument given on p. 92 of [3], $f_{\eta}$ can be extended to an isomorphism $f: p_{1}^{*}\left(\mathscr{C}^{\prime}\right) \widetilde{\rightarrow} p_{2}^{*}\left(\mathscr{C}^{\prime}\right)$ and $\mathscr{C}^{\prime}$ can be descended to a curve $\mathscr{C}$ over $S$.

Moreover, since $S^{\prime} \rightarrow S$ is faithfully flat, (SGA 1, Exposé VIII, Théorème $5.2)$ asserts the exactness of

$$
\operatorname{Hom}_{S}(J(\mathscr{C}), \mathfrak{X}) \longrightarrow \operatorname{Hom}_{S^{\prime}}\left(J\left(\mathscr{C}^{\prime}\right), \mathfrak{X}^{\prime}\right) \underset{p_{2}^{*}}{\stackrel{p_{1}^{*}}{\longrightarrow}} \operatorname{Hom}_{S^{\prime \prime}}\left(J\left(\mathscr{C}^{\prime \prime}\right), \mathfrak{X}^{\prime \prime}\right),
$$

where $\mathscr{C}^{\prime \prime}=\mathscr{C} \times{ }_{S} S^{\prime \prime}$ and $\mathfrak{X}^{\prime \prime}=\mathfrak{X} \times{ }_{S} S^{\prime \prime}$. Since $\phi_{\eta}^{\prime}=\phi_{0} \otimes_{K} K^{\prime},\left(p_{1}^{*} \phi^{\prime}\right)_{\eta}$ becomes equal to $\left(p_{2}^{*} \phi^{\prime}\right)_{\eta}$. Furthermore, every irreducible component of $S^{\prime \prime}$ dominates $S$ (cf. EGA IV ${ }^{2}$, Proposition (2.3.4), (iii)). Therefore, by rigidity (cf. [9], Corollary 6.2), we conclude $p_{1}^{*} \phi^{\prime}=p_{2}^{*} \phi^{\prime}$ and the existence of an isomorphism $\phi: J(\mathscr{C}) \widetilde{X}$ such that $\phi \otimes_{A} A^{\prime}=\phi^{\prime}$ (cf. SGA 1, Expose VIII, Corollaire 5.4). Moreover, by the same rigidity, we can see that $\phi$ gives an isomorphism between $(J(\mathscr{C}), \lambda(\mathscr{C}))$ and $(\mathfrak{X}, \lambda)$.

Q.E.D.

From this proposition, we can easily deduce

Conollary 4.2. Let $A$ be a discrete valuation ring with $k=A / \mathfrak{M}_{A}$ and $K=$ f.f. $(A)$. We denote by $\eta$ and $s$ the generic point and the special point of $S=\operatorname{Spec}(A)$, respectively. Let $(\mathfrak{X}, \lambda)$ be a principally polarized abelian scheme over $S$, and $\theta$ a divisor on $\bar{X}=\mathfrak{X} \otimes_{A} \bar{K}$ such that $\lambda \otimes_{A} \bar{K}=$ $\Lambda\left(\mathcal{O}_{\bar{X}}(\theta)\right)$. Assume the irreducibility of $\theta$. Moreover, let $C$ be a complete non-singular curve rational over $K$ of genus $g \geqq 2$ with an isomorphism

$$
\phi_{0}:(J(C), \lambda(C)) \stackrel{\sim}{\longrightarrow}(\mathfrak{X}, \lambda)_{\eta} .
$$

Then there exist a smooth curve $\mathscr{C}$ over $S$ with connected geometric fibres and with $C$ as the generic fibre, and an isomorphism

$$
\phi:(J(\mathscr{C}), \lambda(\mathscr{C})) \stackrel{\sim}{\longrightarrow}(\mathfrak{X}, \lambda)
$$

such that $\phi_{\eta}=\phi_{0}$.

We shall extend this fact over a regular local ring.

Proposition 4.3. Let $(A, \mathfrak{M})$ be a regular local ring with $k=A / \mathfrak{M}$ and $K=$ f.f. $(A)$. Let $b$ and $a$ be the generic point and the closed point of $S=\operatorname{Spec}(A)$. Let $\mathfrak{X}$ be an abelian scheme over $S$ with a principal 
polarization $\lambda$. Assume that there exists a good curve $C$ over $K$ of genus $\mathrm{g} \geqq 2$ with an isomorphism

$$
(J(C), \lambda(C)) \stackrel{\sim}{\longrightarrow}(\mathfrak{X}, \lambda) \otimes_{A} K .
$$

Then there exists a good curve $C_{0}$ over $k$ such that

$$
\left(J\left(C_{0}\right), \lambda\left(C_{0}\right)\right) \stackrel{\sim}{\longrightarrow}(\mathfrak{X}, \lambda)_{a}
$$

Moreover, if $C$ is non-singular and $\lambda \otimes_{A} \bar{k}=\Lambda\left(\mathcal{O}_{X}(\theta)\right)$ for an irreducible divisor $\theta$ on $\mathfrak{X} \otimes_{A} \bar{k}, C_{0}$ is also non-singular.

Proof. We shall use induction on the dimension of $A$.

If $\operatorname{dim} A=1, A$ is a discrete valuation ring. Therefore, our assertion in this case is just Proposition 4.1 and Corollary 4.2.

Assume $\operatorname{dim} A=n>1$. Then we take a regular parameter $t$ and we put $A_{1}=A_{(t)}$. Let $\left(\mathfrak{X}_{1}, \lambda_{1}\right)=(\mathfrak{X}, \lambda) \otimes_{A} A_{1}$. We denote by $\eta_{1}$ and $s_{1}$ the generic point and the special point of $\operatorname{Spec}\left(A_{1}\right)$, respectively. Then obviously

$$
\left(\mathfrak{X}_{1}, \lambda_{1}\right)_{\eta_{1}} \simeq(\mathfrak{X}, \lambda)_{b} \text {. }
$$

Hence, by virtue of Proposition 4.1, there exists a good curve $C_{1}$ over $A_{1} / t A_{1}=$ f.f. $(A /(t))$ such that

$$
\left(J\left(C_{1}\right), \lambda\left(C_{1}\right)\right) \simeq\left(\mathfrak{X}_{1}, \lambda_{1}\right)_{s_{1}} .
$$

Moreover let $(\overline{\mathfrak{X}}, \bar{\lambda})=(\mathfrak{X}, \lambda) \otimes_{A}(A /(t))$. Then we get

$$
(\overline{\mathfrak{X}}, \bar{\lambda})_{\bar{a}} \simeq\left(\mathfrak{X}_{1}, \lambda_{1}\right)_{s_{1}} \simeq\left(J\left(C_{1}\right), \lambda\left(C_{1}\right)\right)
$$

and

$$
\operatorname{dim}(A /(t))=n-1,
$$

where $\bar{a}$ is the generic point of $\operatorname{Spec}(A /(t))$. Therefore, by induction, we obtain our assertion.

Q.E.D.

The next proposition will play an essential role in the proof of the main theorem in Section 5.

Proposition 4.4. Let $\left(A, \mathfrak{M}_{A}\right)$ be a complete valuation ring with residue field $k$ and f.f. $(A)=K$. Let $K^{\prime}$ be a finite algebraic extension of $K$ and $A^{\prime}$ the integral closure of $A$ in $K^{\prime}$. As is well-known, $A^{\prime}$ is also a complete discrete valuation ring and is a finite free A-module. We put $k^{\prime}=$ 
$A^{\prime} / \mathfrak{M}_{A^{\prime}}, S^{\prime}=\operatorname{Spec}\left(A^{\prime}\right)$ and $S=\operatorname{Spec}(A)$. Let $(\mathfrak{X}, \lambda)$ be a principally polarized abelian scheme over $S, C^{\prime}$ a complete non-singular curve rational over $K^{\prime}$ and $C_{0}$ a complete non-singular non-hyperelliptic curve rational over $k$. Assume there exists an isomorphism

$$
\psi:\left(J\left(C^{\prime}\right), \lambda\left(C^{\prime}\right)\right) \stackrel{\sim}{\longrightarrow}(\mathfrak{X}, \lambda) \otimes_{A} K^{\prime} .
$$

Then, Proposition 4.1 asserts that $C^{\prime}$ has a deformation $\mathscr{C}^{\prime}$ over $S^{\prime}$. Moreover we suppose that

$$
C_{0} \otimes_{k} k^{\prime} \simeq \mathscr{C}_{s^{\prime}}^{\prime}
$$

and

$$
(\mathfrak{X}, \lambda)_{s} \simeq\left(J\left(C_{0}\right), \lambda\left(C_{0}\right)\right),
$$

where $s$ and $s^{\prime}$ are the special points of $S$ and $S^{\prime}$, respectively. Then there exists a smooth curve $\mathscr{C}$ over $S$ with isomorphisms

$$
\phi:(J(\mathscr{C}), \lambda(\mathscr{C})) \stackrel{\sim}{\longrightarrow}(\mathfrak{X}, \lambda)
$$

and

$$
f: \mathscr{C} \times{ }_{S} S^{\prime} \longrightarrow \mathscr{C}^{\prime}
$$

such that $f$ induces $\phi \otimes_{A} A^{\prime}$ and $\phi \otimes_{A} K^{\prime}$ is equal to $\psi$.

Proof. For any non-negative integer $n$, we put $A_{n}=A / \mathfrak{M}_{A}^{n+1}, S_{n}=$ $\operatorname{Spec}\left(A_{n}\right), A_{n}^{\prime}=A^{\prime} / \mathfrak{M}_{A}^{n+1} A^{\prime}$ and $S_{n}^{\prime}=\operatorname{Spec}\left(A_{n}^{\prime}\right)$. Then we have the cartesian product

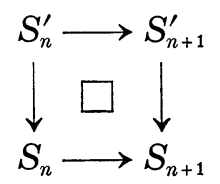

and the following diagram with exact horizontal lines

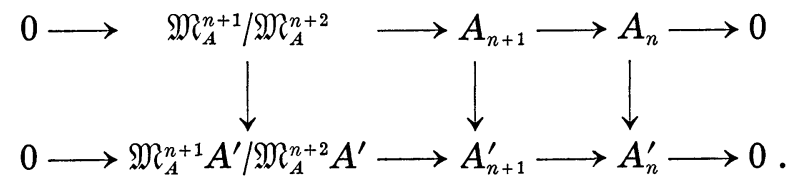

Moreover we put $C_{n}^{\prime}=\mathscr{C}^{\prime} \times{ }_{S^{\prime}} S_{n}^{\prime}, \quad\left(X_{n}, \lambda_{n}\right)=(\mathfrak{X}, \lambda) \times_{s} S_{n}$ and $\left(X_{n}^{\prime}, \lambda_{n}^{\prime}\right)=$ $(\mathfrak{X}, \lambda) \times{ }_{s} S_{n}^{\prime}$. For proving our assertion by induction on $n$, we assume the existence of a curve $C_{n}$ over $S_{n}$ such that 


$$
C_{n} \times_{S_{n}} S_{n}^{\prime} \simeq C_{n}^{\prime} \text { and }\left(J\left(C_{n}\right), \lambda\left(C_{n}\right)\right) \simeq\left(X_{n}, \lambda_{n}\right) .
$$

Then, setting suitably base points of lifting spaces, we obtain the following commutative diagram

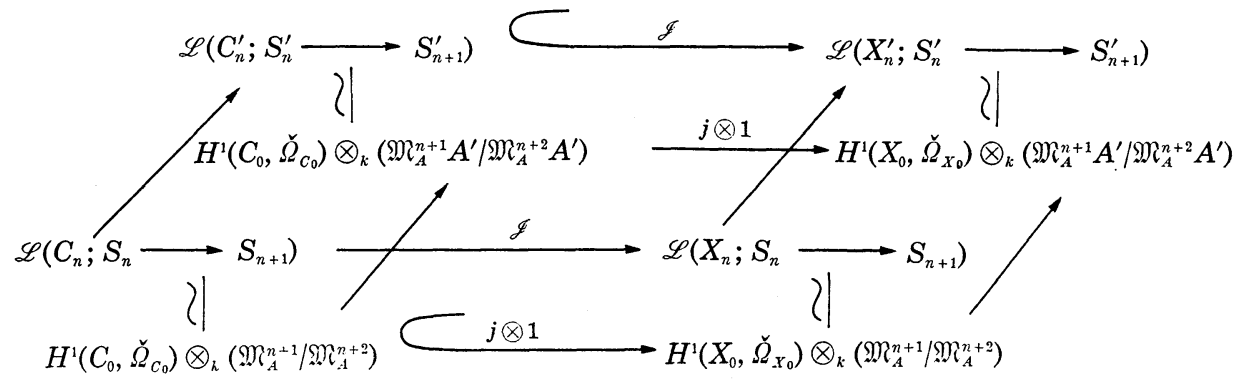

(cf. Propositions 3.1, 3.2 and Lemma 3.3). Obviously, in

$$
\begin{aligned}
& H^{1}\left(X_{0}, \check{\Omega}_{X_{0}}\right) \otimes_{k}\left(\mathfrak{M}_{A}^{n+1} A^{\prime} / \mathfrak{M}_{A}^{n+2} A^{\prime}\right), \\
& \left(H^{1}\left(C_{0}, \check{\Omega}_{C_{0}}\right) \otimes_{k}\left(\mathfrak{M}_{A}^{n+1} A^{\prime} / \mathfrak{M}_{A}^{n+2} A^{\prime}\right)\right) \cap\left(H^{1}\left(X_{0}, \check{\Omega}_{X_{0}}\right) \otimes_{k}\left(\mathfrak{M}_{A}^{n+1} / \mathfrak{M}_{A}^{n+2}\right)\right) \\
& \quad=H^{1}\left(C_{0}, \check{\Omega}_{C_{0}}\right) \otimes\left(\mathfrak{M}_{A}^{n+1} / \mathfrak{M}_{A}^{n+2}\right),
\end{aligned}
$$

that is, in

$$
\begin{aligned}
& \mathscr{L}\left(X_{n}^{\prime} ; S_{n}^{\prime} \longrightarrow S_{n+1}^{\prime}\right), \\
& \mathscr{L}\left(C_{n}^{\prime} ; S_{n}^{\prime} \longrightarrow S_{n+1}^{\prime}\right) \cap \mathscr{L}\left(X_{n} ; S_{n} \longrightarrow S_{n+1}\right)=\mathscr{L}\left(C_{n} ; S_{n} \longrightarrow S_{n+1}\right) .
\end{aligned}
$$

Hence, there exists a curve $C_{n+1}$ over $S_{n+1}$ such that

$$
C_{n+1} \times_{S_{n+1}} S_{n} \simeq C_{n}, \quad C_{n+1} \times_{S_{n+1}} S_{n+1}^{\prime} \simeq C_{n+1}^{\prime}
$$

and

$$
\left(J\left(C_{n+1}\right), \lambda\left(C_{n+1}\right)\right) \simeq\left(X_{n+1}, \lambda_{n+1}\right) .
$$

Thus we obtain a system of curves $\left\{C_{n} / S_{n}\right\}$ and it determines a curve $\mathscr{C}$ over $S$ satisfying our requiring conditions.

Q.E.D.

CoRollary 4.5. Let $\left(A, \mathfrak{M}_{A}\right)$ be a discrete valuation ring with $k=$ $A / \mathfrak{M}_{A}$ and $K=$ f.f. $(A)$. We assume that $A$ is a Nagata ring. Let $L$ be a finite algebraic extension of $K$ and $B$ a discrete valuation ring for $L$ over $A$ with $\ell=B / \mathfrak{M}_{B}$. Let $k^{\prime}$ be a finitely generated field over $k$ in which the algebraic closure of $k$ is separable over $k$, and $\ell^{\prime}$ a field containing $k^{\prime}$ and such that $\ell^{\prime}=k^{\prime} \ell$. 


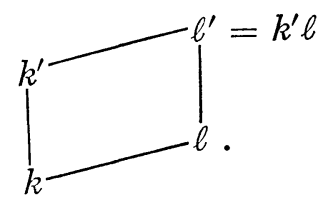

Let $(\mathfrak{X}, \lambda)$ be a principally polarized abelian scheme over $\operatorname{Spec}(A), C^{\prime}$ a complete non-singular curve rational over $L$ and $C_{0}$ a complete nonsingular non-hyperelliptic curve rational over $k^{\prime}$. Assume there exists an isomorphism

$$
\psi:\left(J\left(C^{\prime}\right), \lambda\left(C^{\prime}\right)\right) \longrightarrow(\mathfrak{X}, \lambda) \otimes_{A} L,
$$

and we denote by $\mathscr{C}^{\prime}$ a deformation of $C^{\prime}$ over $\operatorname{Spec}(B)$ given by Proposition 4.1. We put

$$
C_{0}^{\prime}=\mathscr{C}^{\prime} \otimes_{B} \ell
$$

Moreover, we suppose that

$$
C_{0} \otimes_{k^{\prime}} \ell^{\prime} \simeq C_{s}^{\prime} \otimes_{\ell} \ell^{\prime}
$$

and

$$
(\mathfrak{X}, \lambda) \otimes_{A} k^{\prime} \simeq\left(J\left(C_{0}\right), \lambda\left(C_{0}\right)\right) .
$$

Then there exist a finitely generated field $K^{\prime}$ over $K$ in which the algebraic closure of $K$ is separable over $K$, and a curve $C$ rational over $K^{\prime}$ such that if we denote by $L^{\prime}$ a field containing $K^{\prime}$ and $L$ and satisfying $L^{\prime}=K^{\prime} L$,

$$
C^{\prime} \otimes_{L} L^{\prime} \simeq C \otimes_{K^{\prime}} L^{\prime}
$$

and

$$
(J(C), \lambda(C)) \simeq(X, \lambda) \otimes_{A} K^{\prime} \text {. }
$$

Proof. By virtue of Proposition 1.3, there exist finitely generated fields $K^{\prime}$ over $K$ and $L^{\prime}$ over $L$; and discrete valuation rings $\left(A^{\prime}, \mathfrak{M}_{A^{\prime}}\right)$ for $K^{\prime}$ and $\left(B^{\prime}, \mathfrak{M}_{B^{\prime}}\right)$ for $L^{\prime}$ with residue fields $k^{\prime}$ and $\ell^{\prime \prime}$, respectively, such that $B^{\prime}>A^{\prime}>A, B^{\prime}>B, \mathfrak{M}_{A^{\prime}}=\mathfrak{M}_{A} A^{\prime}, L^{\prime}=K^{\prime} L, \ell^{\prime \prime}$ is a finite algebraic extension of $\ell^{\prime}$ and the algebraic closure of $K$ in $K^{\prime}$ is separable over $K$ :
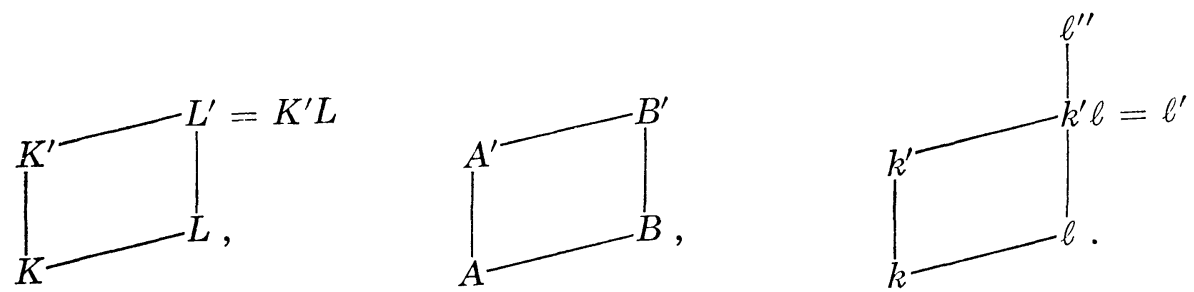

Put 


$$
\left(\mathfrak{X}^{*}, \lambda^{*}\right)=(\mathfrak{X}, \lambda) \otimes_{A} \hat{A}^{\prime} \quad \text { and } \quad C^{*}=C^{\prime} \otimes_{L} \hat{L}^{\prime},
$$

where the symbol ^ means the completion. Then we have the isomorphisms

$$
\begin{aligned}
& \left(J\left(C^{*}\right),\left(C^{*}\right)\right) \simeq\left(\mathfrak{X}^{*}, \lambda^{*}\right) \otimes_{\hat{A}^{\prime}} \hat{L}^{\prime}, \\
& C_{0} \otimes_{k^{\prime}} \ell^{\prime \prime} \simeq\left(C_{0} \otimes_{k^{\prime}} \ell^{\prime}\right) \otimes_{\ell^{\prime}} \ell^{\prime \prime} \simeq\left(C_{0}^{\prime} \otimes_{\ell} \ell^{\prime}\right) \otimes_{\ell^{\prime}} \ell^{\prime \prime} \simeq C_{0}^{\prime} \otimes_{\ell} \ell^{\prime \prime}
\end{aligned}
$$

and

$$
\left(J\left(C_{0}\right), \lambda\left(C_{0}\right)\right) \simeq(\mathfrak{X}, \lambda) \otimes_{A} k^{\prime} \simeq\left(\mathfrak{X}^{*}, \lambda^{*}\right) \otimes_{\hat{A}} k^{\prime} .
$$

Hence, in view of Proposition 4.4, there exists a curve $C$ rational over $\hat{K}^{\prime}$ such that

$$
(J(C), \lambda(C)) \simeq\left(\mathfrak{X}^{*}, \lambda^{*}\right) \otimes_{\hat{A}^{\prime}} \hat{K}^{\prime}
$$

and

$$
C \otimes_{\hat{R}^{\prime}} \hat{L}^{\prime} \simeq C^{* *}
$$

Here we can choose a finitely generated subfield $K^{\prime \prime}$ in $\hat{K}^{\prime}$ over $K^{\prime}$ so that

$$
C \text { is rational over } K^{\prime \prime}, \quad(J(C), \lambda(C)) \simeq(\mathfrak{X}, \lambda) \otimes_{A} K^{\prime \prime} .
$$

and putting $L^{\prime \prime}=K^{\prime \prime} L$,

$$
C \otimes_{K^{\prime \prime}} L^{\prime \prime} \simeq C^{\prime} \otimes_{L} L^{\prime \prime}
$$

Therefore, noticing Lemma 1.4, we can see that these $C, L^{\prime \prime}$ and $K^{\prime \prime}$ are just ones we are requiring.

Q.E.D.

\section{§5. The fields of moduli for non-hyperelliptic curves}

Under the preliminaries in the preceding sections, we shall prove the following main theorem.

THEOREM 5.1. Let $C$ be a non-hyperelliptic complete non-singular curve of genus g rational over a field of characteristic $p>0$. Then the field of moduli for $C$ coincides with that for $(J(C), \lambda(C))$.

Proof. First we notice that the field of moduli $k_{C}$ for $C$ contains the field of moduli $k_{(J(C), \lambda(C))}$ for $(J(C), \lambda(C))$. Hence it is sufficient to show that $k_{(J(C), \lambda(C))}$ contains $k_{C}$.

Let $(X, \lambda)$ be a principally polarized abelian variety over $K$ such that $(X, \lambda) \sim(J(C), \lambda(C))$, where $\sim$ is the geometric isomorphism. Then we may assume that $C$ is rational over a finite algebraic extension $L$ over $K$. Now let $H_{g, 1,1}$ be the fine moduli space of principally polarized abelian 
schemes with linear rigidifications (cf. [9], Chap. 7, § 2). Fixing a linear rigidification suitably, $(X, \lambda)$ defines a $K$-rational point $x$ on $H_{g, 1,1}$. We have no loss of generality even if we assume $K=k(x)$. Let $T$ be the locus of $x$ in $H_{g, 1,1}$, and $(Z, \Lambda)$ the universal abelian scheme over $T$. Then, of course, we have

$$
(Z, \Lambda)_{x} \simeq(X, \lambda) .
$$

If we choose a suitable faithfully flat extension $T^{\prime} \rightarrow T$ (cf. Appendix, Corollary A2), then there exists a relative Cartier divisor $\theta$ on $Z^{\prime}=$ $Z \times_{T} T^{\prime}$ such that

$$
\Lambda\left(\mathcal{O}_{Z^{\prime}}(\theta)\right)=\Lambda \times_{T} T^{\prime}
$$

Since, for a point $x^{\prime}$ in $T^{\prime}$ lying over $x, \theta_{x^{\prime}}$ is geometrically integral, the set

$$
U^{\prime}=\left\{t^{\prime} \in T^{\prime} \mid \theta_{t^{\prime}} \text { is geometrically integral }\right\}
$$

is non-empty open subset in $T^{\prime}$ (cf. EGA IV ${ }^{3}$, Théorème (12.2.4), (viii)). Therefore, the set

$$
\left\{\left\{t^{\prime} \in T^{\prime} \mid(Z, \Lambda)_{t^{\prime}} \sim \begin{array}{l}
\text { the polarized jacobian variety of a } \\
\text { non-hyperelliptic curve }
\end{array}\right\}\right.
$$

is non-empty open in $T^{\prime}$. Hence, noticing that $T^{\prime} \rightarrow T$ is an open map, there exists a closed simple point $t$ of $T$ such that $(Z, \Lambda)_{t}$ is geometrically isomorphic to the polarized jabocian variety of a non-hyperelliptic curve. Here, we put $A=\mathcal{O}_{T, t}$. Then $A$ is a regular local ring with

$$
A / \mathfrak{M}_{A}=F_{q} \quad \text { for some } q=p^{n}
$$

and

$$
\text { f.f. }(A)=k(T)=k(x)=K
$$

Furthermore we put

$$
(\mathfrak{X}, \Lambda)=(Z, \Lambda) \times{ }_{T} \operatorname{Spec}(A) .
$$

Let $\left(t_{1}, \cdots, t_{r}\right)$ be a complete system of regular parameters of $A$, and we put

$$
\begin{aligned}
& A^{(r)}=A_{\left(t_{r}\right)}, \quad A_{r}=A /\left(t_{r}\right), \quad \text { f.f. }\left(A_{r}\right)=K_{r}, \\
& A^{(r-1)}=\left(A_{r}\right)_{\left(t_{r-1}\right)}, \quad A_{r-1}=A_{r} /\left(t_{r-1}\right)=A /\left(t_{r}, t_{r-1}\right), \quad \text { f.f. }\left(A_{r-1}\right)=K_{r-1}, \\
& A^{(1)}=A_{2\left(t_{1}\right)}, \quad A_{1}=A_{2} /\left(t_{1}\right)=A /\left(t_{r}, \cdots, t_{1}\right)=F_{q}, \quad \text { f.f. }\left(A_{1}\right)=K_{1}=F_{q} .
\end{aligned}
$$


Note that these $A^{(i)}$ 's are discrete valuation rings. Put

$$
\left(\mathfrak{X}_{i}, \Lambda_{i}\right)=(\mathfrak{X}, \Lambda) \otimes_{A} A^{(i)}
$$

and

$$
\left(X_{i}, \lambda_{i}\right)=(\mathfrak{X}, \Lambda) \otimes_{A} K_{i}
$$

for each $i=1,2, \cdots, r$. Then we obtain the following chain of deformations:

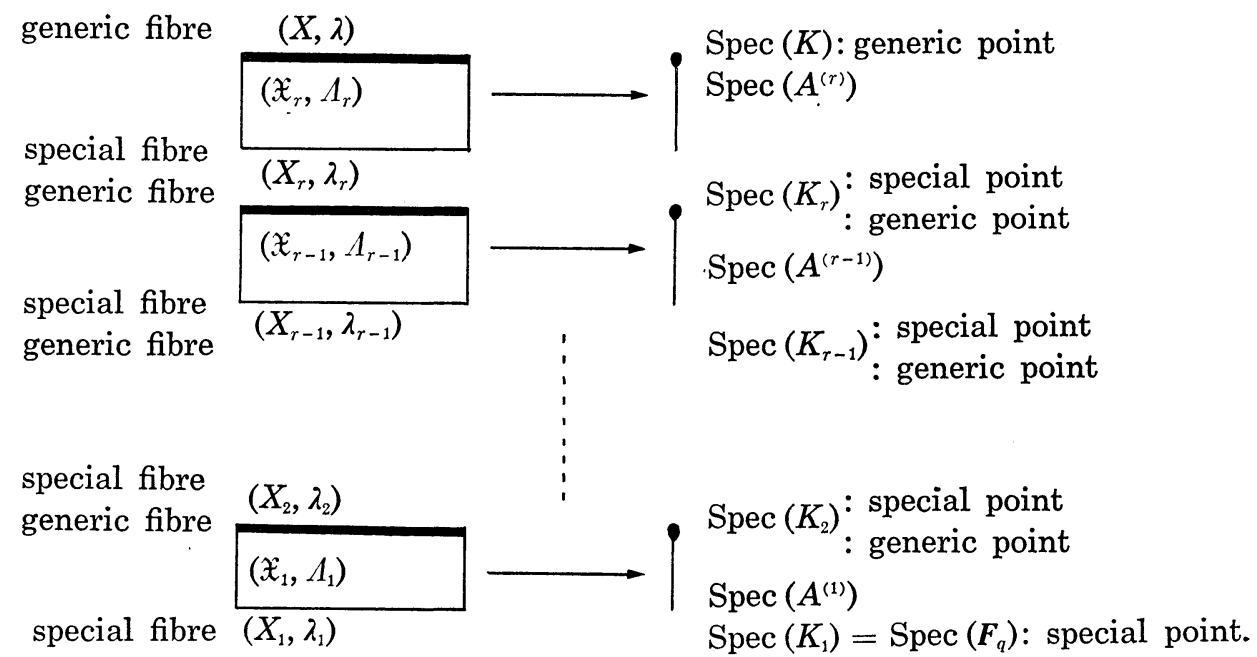

Let $B^{(r)}$ be the localization at a maximal ideal of the integral closure of $A^{(r)}$ in $L, L_{r}=B^{(r)} / \mathfrak{M}_{B^{(r)}}, B^{(r-1)}$ be the localization at a maximal ideal of the integral closure of $A^{(r-1)}$ in $L_{r}, L_{r-1}=B^{(r-1)} / \mathfrak{M}_{B^{(r-1)}}$ and so on. Thus we can define fields and discrete valuation rings

$$
L_{r}, L_{r-1}, \cdots, L_{1} ; \quad B^{(r)}, B^{(r-1)}, \cdots, B^{(1)} .
$$

Furthermore, by virtue of Corollary 4.2, there exist one by one smooth curves

$$
\mathscr{C}_{r} \text { over } \operatorname{Spec}\left(B^{(r)}\right), \quad \mathscr{C}_{r-1} \text { over } \operatorname{Spec}\left(B^{(r-1)}\right), \cdots, \quad \mathscr{C}_{1} \text { over } \operatorname{Spec}\left(B^{(1)}\right)
$$

such that

$$
\begin{aligned}
& \mathscr{C}_{r} \otimes_{B^{(r)}} L \simeq C, \quad \mathscr{C}_{r} \otimes_{B^{(r)}} L_{r} \simeq \mathscr{C}_{r-1} \otimes_{B^{(r-1)}} L_{r}, \cdots, \\
& \mathscr{C}_{2} \otimes_{B^{(2)}} L_{2} \simeq \mathscr{C}_{1} \otimes_{B^{(1)}} L_{2}
\end{aligned}
$$

and 


$$
\left(J\left(\mathscr{C}_{r}\right), \lambda\left(\mathscr{C}_{r}\right)\right) \simeq\left(\mathfrak{X}_{r}, \Lambda_{r}\right) \otimes_{A^{(r)}} B^{(r)}, \cdots,\left(J\left(\mathscr{C}_{1}\right), \lambda\left(\mathscr{C}_{1}\right)\right) \simeq\left(\mathfrak{X}_{1}, A_{1}\right) \otimes_{A^{(1)}} B^{(1)} .
$$

Hence, putting $\mathscr{C}_{i} \otimes_{B^{(i)}} L_{i}=C_{i}(i=1,2, \cdots, r)$, we obtain the following chain of deformations

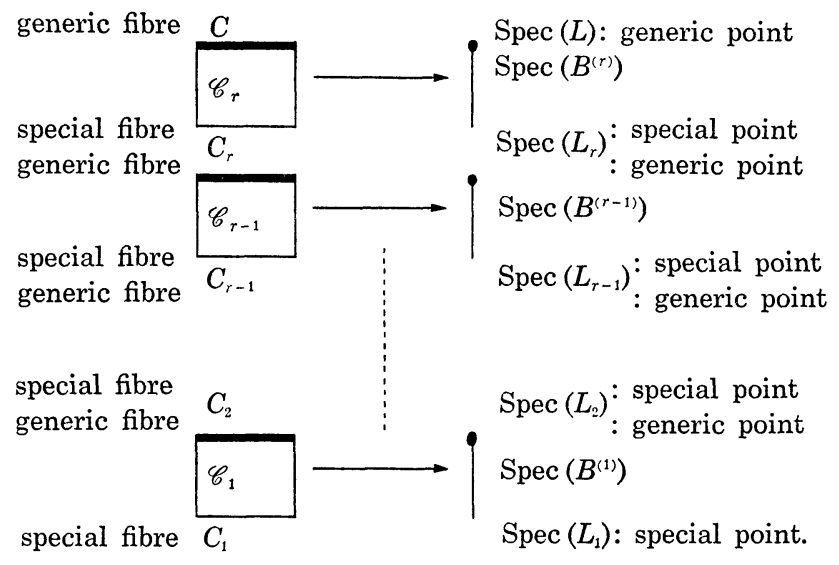

Applying Corollary 4.5 to $C_{1},\left(\mathfrak{X}_{1}, \Lambda_{1}\right)$ and the separable extension $L_{1} / K_{1}$, we have a finitely generated field $K_{2}^{\prime}$ over $K_{2}$ in which the algebraic closure of $K_{2}$ is separable over $K_{2}$, and a curve $C_{2}^{\prime}$ rational over $K_{2}^{\prime}$ such that if we denote by $L_{2}^{\prime}$ a field containing $K_{2}^{\prime}$ and $L_{2}$ and satisfying $L_{2}^{\prime}=$ $K_{2}^{\prime} L_{2}$,

$$
C_{2} \otimes_{L_{2}} L_{2}^{\prime} \simeq C_{2}^{\prime} \otimes_{K_{2}^{\prime}} L_{2}^{\prime}
$$

and

$$
\left(J\left(C_{2}^{\prime}\right), \lambda\left(C_{2}^{\prime}\right)\right) \simeq\left(\mathfrak{X}_{2}, \Lambda_{2}\right) \otimes_{A_{2}} K_{2}^{\prime} .
$$

Applying again Corollary 4.5 to $\mathscr{C}_{2},\left(\mathfrak{X}_{2}, \Lambda_{2}\right)$ and the extension $K_{2}^{\prime} / K_{2}$, we get a finitely generated field $K_{3}^{\prime}$ over $K_{3}$ and a curve $C_{3}^{\prime}$ rational over $K_{3}^{\prime}$ satisfying the similar properties. Proceeding with these steps, we obtain finally a finitely generated field $K^{\prime}$ over $K$ in which the algebraic closure of $K$ is separable over $K$, and a curve $C^{\prime}$ rational over $K^{\prime}$ such that

$$
\left(J\left(C^{\prime}\right), \lambda\left(C^{\prime}\right)\right) \simeq(X, \lambda) \otimes_{K} K^{\prime} .
$$

Since $C \sim C^{\prime}$, the field of moduli $k_{C}$ for $C$ is contained in $L \cap K^{\prime}$. Moreover, from the construction of $K^{\prime}, L \cap K^{\prime}$ is separable over $K$. Let $M$ be a normal extension over $K$ containing $L$, and we put $C^{\prime \prime}=C \otimes_{L} M$. Then, by virtue of Torelli's theorem, 


$$
C^{\prime \prime \sigma} \sim C^{\prime \prime} \sim C \quad \text { for any } \sigma \in \operatorname{Aut}(M / K) \text {. }
$$

Hence, by Fm 2) in Definition 2.2, we conclude the inclusion

$$
K \supset k_{C} \text {. }
$$

Thus we obtain the converse relation

$$
k_{(J(C), \lambda(C))} \supset k_{C},
$$

and we are done.

Q.E.D.

\section{\$6. The fields of rationality for curves of genus 3}

In Section 5, we have devoted to show the coincidence of the fields of moduli for a non-hyperelliptic curve of any genus and of its jacobian variety, but if we restrict ourselves to the curves of genus 3 , we can show the coincidence of fields of rationality for a curve and for its polarized jacobian variety.

For proving this fact, we shall start with an easy result of descent theory.

Proposition 6.1. Let ( $\subseteq, Q)$ be an FM-system given by Theorem 2.3, Theorem 2.4 or etc. Let $Q$ and $Q^{\prime}$ be two objects in $\subseteq$, and $\lambda: Q \rightarrow Q^{\prime} a$ geometric isomorphism. Suppose that $Q$ has no geometric automorphisms other than the identity map. Then $\lambda$ is rational over any field of rationality for $Q$ and $Q^{\prime}$.

(cf. [13], Proposition 2.)

Proposition 6.2. Let $C$ be a complete non-singular curve over a field of characteristic 0. Suppose C has no geometric automorphisms other than the identity. Then we have

$$
\mathrm{FR}(C)=\mathrm{FR}((J(C), \lambda(C)) .
$$

Proof. The inclusion $\subset$ is obvious.

Conversely, let $k_{0}$ be a field contained in FR $((J(C), \lambda(C)))$. Then we may assume that $C$ is rational over a field $k$ which is a Galois extension over $k_{0}$ with Galois group $G$. For any $\sigma \in G$,

$$
\left(J\left(C^{\sigma}\right), \lambda\left(C^{\sigma}\right)\right) \cong(J(C), \lambda(C)) .
$$

Hence, by Torelli's theorem, there exists a geometric isomorphism

$$
\lambda_{\sigma}: C \longrightarrow C^{\circ} \text {. }
$$


By Proposition 6.1, $\lambda_{\sigma}$ is defined over $k$. Moreover, by our assumption,

$$
\left(\lambda_{\tau}^{\sigma} \circ \lambda_{\sigma}\right)^{-1} \circ \lambda_{\tau \sigma}=\mathbf{1}_{C}
$$

and

$$
\lambda_{\tau \sigma}=\lambda_{\tau}^{\sigma} \circ \lambda_{\sigma}
$$

for any $\sigma, \tau \in G$. Therefore, by the descent theory, there exists a curve $C_{0}$ rational over $k_{0}$ such that

$$
C_{0} \otimes_{k_{0}} k \simeq C
$$

ThEOREM 6.3. Let $(X, \lambda)$ be a principally polarized abelian variety over a field $k$ of dimension 3. Then there exists a good curve $C$ over $k$ such that

$$
(J(C), \lambda(C)) \simeq(X, \lambda) .
$$

Proof. Let $H_{3,1,1}$ be the fine moduli space of principally polarized abelian schemes with linear rigidifications. Since the local moduli functor for an abelian variety is formally smooth (cf. [10], Theorem 2.4.1), $H_{3,1,1}$ is smooth over Spec $(\mathbf{Z})$. Hence every point of $H_{3,1,1}$ is regular.

Now choosing a linear rigidification $\phi$ of $(X, \lambda),(X, \lambda, \phi)$ defines a $k$-rational point $\alpha$ of $H_{3,1,1}$. Then $k$ contains $k(\alpha)$. Replacing $k$ by $k(\alpha)$, if necessary, we may assume that $k=k(\alpha)$. We denote by $A$ the regular local ring $\mathcal{O}_{H_{3,1,1}, \alpha}$. Then the canonical $A$-valued point of $H_{3,1,1}$ defines a principally polarized abelian scheme

$$
(\mathfrak{X}, \Lambda) \text { over } \operatorname{Spec}(A)
$$

with $(\mathfrak{X}, \Lambda) \otimes_{A} k \simeq(X, \lambda)$. Let $b$ and $a$ be the generic point and the special point of $\operatorname{Spec}(A)$, respectively. By the main theorem of [11],

$$
(\mathfrak{X}, \Lambda)_{b} \sim \text { the polarized jacobian variety of a curve. }
$$

On the other hand, since $(\mathfrak{X}, \Lambda)_{b}$ is a generic polarized abelian variety over a field of characteristic 0 , the curve corresponding to it has no nontrivial automorphism. Hence, by virtue of Proposition 6.2, there exists a curve $C^{\prime}$ rational over $K=$ f.f. $(A)$ such that

$$
\left(J\left(C^{\prime}\right), \lambda\left(C^{\prime}\right)\right) \simeq(\mathfrak{X}, \Lambda)_{b} .
$$

Therefore, in view of Proposition 4.3, there exists a good curve $C_{0}$ over $k$ such that

$$
\left(J\left(C_{0}\right), \lambda\left(C_{0}\right)\right) \simeq(\mathfrak{X}, \Lambda)_{a} \simeq(X, \Lambda) .
$$




\section{Appendix}

By [7], Proposition 3.1, Koizumi shows the fact:

Let $V$ be the underlying variety of an abelian variety, and it be rational over a field $K$ of characteristic $\neq 2$. Then every algebraic equivalence class of divisors on $V$ has a representative rational over a separable algebraic extension of $K$.

This fact is one of the conditions guaranteeing the existence of the fields of moduli for abelian varieties with $\mathscr{P}$-structures (cf. [7], Theorem 3.2).

In the appendix, we shall give another proof of the above statement, which is available even for characteristic two.

Let $S$ be a locally noetherian scheme and $\mathfrak{X}$ a projective abelian scheme over $S$. Then, Grothendieck's results [4] assert the existence of a scheme $\operatorname{Hom}(\mathfrak{X}, \hat{X})$ representing the family of all homomorphisms from $\mathfrak{X}$ to $\hat{X}$. Let $\Lambda: \operatorname{Pic}(\mathfrak{X}) \rightarrow \operatorname{Hom}_{\mathcal{S}}(\mathfrak{X}, \hat{X})$ be the morphism assigning $\Lambda(\mathscr{L})$ to an invertible sheaf $\mathscr{L}$ on $\mathfrak{X}$. Under these notation, we have

Proposition A1. $\Lambda: \operatorname{Pic}(\mathfrak{X}) \rightarrow \operatorname{Hom}_{s}(\mathfrak{X}, \hat{\mathfrak{X}})$ is smooth.

Proof. Let $(A, \mathfrak{M})$ be any artinian local ring and $I$ an ideal of $A$ such that $\mathfrak{M} I=(0)$. Let $t$ be a given $A$-valued point of $\operatorname{Hom}_{s}(\mathfrak{X}, \hat{\mathfrak{X}})$ and $u_{0}$ an $A / I$-valued point of Pic $(\mathfrak{X})$ such that $\Lambda\left(u_{0}\right)=\left.t\right|_{\text {spec }(A / I)}$. Then there exist a homomorphism $\phi: \mathfrak{X} \times{ }_{S} \operatorname{Spec}(A) \rightarrow \hat{X} \times{ }_{s} \operatorname{Spec}(A)$ and an invertible sheaf $L_{0}$ on $\mathfrak{X} \times{ }_{S} \operatorname{Spec}(A / I)$ corresponding to the points $t$ and $u_{0}$, respectively. Since $\Lambda\left(L_{0}\right)=\phi \otimes_{A}(A / I)$, there exists an invertible sheaf $L^{\prime}$ on $\mathfrak{X} \times{ }_{S} \operatorname{Spec}(A)$ such that $\Lambda\left(L^{\prime}\right)=\phi$ (cf. [10], Lemma 2.3.2). So $\left.L^{\prime}\right|_{\mathfrak{X} \times S \text { Spec (A/I) }}$ and $L_{0}$ differ possibly only by an invertible sheaf algebraically equivalent to zero; that is, there exists an $A / I$-valued point $\alpha_{0}$ of $\hat{X}$ such that

$$
\left.L^{\prime}\right|_{X_{S} \operatorname{Spec}(A / I)} \otimes \mathscr{P}_{\alpha_{0}} \simeq L_{0},
$$

where $\mathscr{P}$ is the Poincaré invertible sheaf on $\mathfrak{X} \times_{s} \hat{\mathbb{X}}$. Since $\hat{\mathfrak{X}}$ is smooth over $S, \alpha_{0}$ can be extended to an $A$-valued point $\alpha$ of $\hat{\mathbb{X}}$. Then the invertible sheaf $L=L^{\prime} \otimes \mathscr{P}_{\alpha}$ satisfies the condition:

$$
\left.L\right|_{\mathfrak{x} \times S \operatorname{Spec}(A / I)} \simeq L_{0}
$$

and

$$
\Lambda(L)=\phi \text {. }
$$

Therefore, the $A$-valued point $u$ of Pic $(\mathfrak{X})$ corresponding to $L$ is an extension of $u_{0}$ over $t$; that is, this implies the smoothness of $\Lambda$. Q.E.D. 
Conollary A2. For a symmetric homomorphism $\phi: \mathfrak{X} \rightarrow \hat{\mathfrak{X}}$, there exist a faithfully flat extension $S^{\prime} \rightarrow S$ of $S$ and an invertible sheaf $\mathscr{L}$ on $\mathfrak{X} \times{ }_{S} S^{\prime}$ such that $\Lambda(\mathscr{L})=\phi \times{ }_{s} S^{\prime}$.

Proof. Let $x$ be the $S$-valued point of $\operatorname{Hom}_{S}(\mathfrak{X}, \hat{\mathfrak{X}})$ corresponding to $\phi$. Then, by the above proposition, $\Lambda^{-1}(x) \rightarrow S$ is smooth, a fortiori, faithfully flat. Therefore, putting $S^{\prime}=\Lambda^{-1}(x)$ and $x^{\prime}=$ the $S^{\prime}$-valued point of $\mathbf{H o m}_{S}(\mathfrak{X}, \hat{\mathfrak{X}})$ corresponding to $\phi \times{ }_{S} S^{\prime}, \Lambda^{-1}\left(x^{\prime}\right)=\Lambda^{-1}(x) \times{ }_{S} S^{\prime}$ has a crosssection $s$ over $S^{\prime}$. Obviously the invertible sheaf $\mathscr{L}$ corresponding to this cross-section $s$ satisfies our requiring property. Q.E.D.

Proposition A3. Let $X$ be an abelian variety over a field of any characteristic and $V$ the underlying variety of $X$, rational over a field $k$. Then every algebraic equivalence class of divisors on $V$ has a representative rational over a separable algebraic extension of $k$.

Proof. We denote by $\bar{k}$ the algebraic closure of $k$, and we put $\bar{X}=$ $X \otimes_{k} \bar{k}$ and $\bar{V}=V \otimes_{k} \bar{k}$. Let $\bar{L}$ be any invertible sheaf on $\bar{V}$. Our aim is to show the existence of an invertible sheaf $L$ rational over a separable algebraic extension of $k$ such that $\Lambda\left(L \otimes_{k} \bar{k}\right)=\Lambda(\bar{L})$. As is well-known, $X$ is rational over a separable algebraic extension of $k$. Therefore, by virtue of Chow's theorem, there is a separable extension $k^{\prime}$ of $k$ and a homomorphism $\phi: X \otimes_{k} k^{\prime} \rightarrow X \otimes_{k} k^{\prime}$ such that $\phi \otimes_{k^{\prime}}, \bar{k}=\Lambda(\bar{L})$. On the other hand, in view of Proposition A1, $\Lambda^{-1}(\phi)$ is smooth over $k^{\prime}$. Hence there exists a closed point $x$ of $\Lambda^{-1}(\phi)$ whose residue field $k(x)$ is separable over $k^{\prime}$ (cf. EGA IV ${ }^{4}$, Remarque (17.15.10), (iii)). Thus the invertible sheaf corresponding to $x$ satisfies our requiring properties.

Q.E.D.

Addendum. The author is very grateful to Prof. T. Oda for having pointed out the existence of the latest result of Oort and Steenbrink:

F. Oort and J. Steenbrink, The local Torelli problem for algebraic curves. In: J. de geometrie algebrique d'Angers 1979, edited by A. Beaubille; Sijthoff-Noordhoff 1980.

After the present paper was finished, the author found that the main theorem (Theorem 5.1) could be proved more easily in much more general style by using the above Oort-Steenbrink's result. As for the new proof, he will explain in a forthcoming paper. 


\section{REFERENCES}

[1] N. Bourbaki, Algébre, Eléments de Math. 23, Hermann, Paris, 1958.

[2] - Algébre commutative, Eléments de Math. 30, Hermann, Paris, 1964.

[ 3 ] P. Deligne and D. Mumford, The irreducibility of the space of curves of given genus, Publ. Math., 36 (Volume dedicated to O. Zariski), I.H.E.S. (1969), 75-109.

[4] A. Grothendieck, Fondements de la géométrie algébrique, Séminaire Bourbaki 1957-62, Secrétariat Math., Paris (1962).

[ 5 ] — Eléments de géométrie algébrique (with J. Dieudonné), Publ. Math. I.H.E.S., 1960-1967. Refered to as EGA.

[6] A. Grothendieck et al., Séminaire de géométrie algébrique 1, Lecture notes in 224, Springer-Verlag, Heidelberg, 1971. Refered to as SGA 1.

[7] S. Koizumi, The fields of moduli for polarized abelian varieties and for curves, Nagoya Math. J., 48 (1972), 37-55.

[ 8 ] T. Matsusaka, Polarized varieties, fields of moduli and generalized Kummer varieties of polarized abelian varieties, Amer. J. Math., 80 (1958), 45-82.

[ 9 ] D. Mumford, Geometric invariant theory, Ergebnisse, Springer-Verlag, Heidelberg, 1965.

[10] F. Oort, Finite group schemes, local moduli for abelian varieties and lifting problems, algebraic geometry, Oslo 1970, Proceedings of the $5^{\text {th }}$ Nordic SummerSchool in Math., edited by F. Oort.

[11] F. Oort and K. Ueno, Principally polarized abelian varieties of dimension two or three are Jacobian varieties, J. Fac. Sci. Univ. Tokyo, Section IA, Math., 20 (1973), 377-381.

[12] G. Shimura, On the theory of automorphic functions, Ann. of Math., 76 (1959), 101-144.

[13] — On the field of rationality for an abelian variety, Nagoya Math. J., 45 (1972), 167-178.

Department of Mathematics

Faculty of Science and Engineering

Chuo University

Kasuga, Bunkyo-ku

Tokyo, Japan 